\title{
Meta-analysis on the effect of combined exercise program intervention on Korean middle-aged women
}

\author{
Dal-Com Moon', Min-Jeong Kim ${ }^{1, *}$, Chung-Moo Lee ${ }^{2}$ \\ ${ }^{1}$ Global Sport Science, Hankuk University of Foreign Studies, Seoul, Korea \\ ${ }^{2}$ Department of Physical Education, Sookmyung Women's University, Seoul, Korea
}

The purpose of this study is to present a basis of application of an exercise program through a systematic analysis of the effect of combined exercise program for middle-aged women using meta-analysis. It appears that the overall effect size $(0.606)$ of the combined exercise program was positive for improving physical strength and physiological function. The physiologically related variables showed an effect size which is equal to or greater than a middle level. This may be an objective basis to prove that the combined exercise program is an effective intervention method to overcome loss of functions and weakened functions by improving physical strength and preventing reduction of hormones. The combined exercise program was found to be most effective when it was conducted two times a week; for over $1 \mathrm{hr}$ and $30 \mathrm{~min}$ to 2 $\mathrm{hr}$; and at intensity of $40 \%$ or more for an 8-week period. As the combined exercise program showed an effect of a middle level or higher for all the middle-aged women in their age of 40 to 65 , it appeared that this exercise program is very effective for middle-aged women. The results of this study suggest that the combined exercise program for middle-aged women is a very effective intervention method. This study can reduce inefficiencies of overly redundant studies regarding an effect of an exercise program, and can be grounds for the basic work for the integrated classification of the physiological variables.

Keywords: Combined exercise program, Middle-aged women, Meta-analysis, Hormone

\section{INTRODUCTION}

The body of a woman achieves the highest developmental status around the age of 20, and then gradually declines around the middle age, showing an explicit change. Aging is prominent after the age of 40. Physical and physiological changes are manifested such as decrease in basal metabolic rate, aging of cells, and decline in functions and production of immune substances (Bruunsgaard and Pedersen, 2000). This is due to the lack of exercise and nutrition; and decline in function of the endocrine system, resulting in the overall degenerative process of the body, and the rapid decline in functions of various parts of the body. As a result, body weakness in middle-aged women causes various diseases and threatens health in middle-age women (Orsini et al., 2006).

It is well known that lowered physical function can change to various desirable results such as delayed aging and improved physical strength through regular exercises (Huang and Osness, 2005). In particular, regular exercise has been recommended as a method for increasing metabolic rate due to increased muscle mass (Brehm, 1988; Poehlman et al., 1991), decreasing blood pressure (Kiefer et al., 1998), improving insulin resistance (Dwarakanathan, 2006), and improving immune function. Through exercises, production of endocrine hormone grows, and this will result in improvement of immune system and activation of lymphocyte proliferation (Bruunsgaard and Pedersen, 2000; Gleeson, 2000; Lakier Smith, 2003). Therefore, active physical activities and regular exercise of middle-aged women are absolutely necessary to be in a healthy state and it is essential to overcome malfunction and weakness of body functions. In particular, the effect of combined exercise which integrates aerobic exercise and resistance exercise is positive on body composition and lipid metabolism positively (Andersen et al., 1995) and combined exercise

\footnotetext{
${ }^{*}$ Corresponding author: Min-Jeong Kim (D) https://orcid.org/0000-0002-0180-2617 Global Sport Science, Hankuk University of Foreign Studies, 107 Imun-ro, Dongdaemun-gu, Seoul 02450, Korea

Tel: +82-2-2173-3144, Fax: +82-2-2173-3070, E-mail: mj8803@hufs.ac.kr Received: March 25, 2018 / Accepted: May 14, 2018
}

This is an Open Access article distributed under the terms of the Creative Commons Attribution Non-Commercial License (http://creativecommons.org/licenses/by-nc/4.0/) which permits unrestricted non-commercial use, distribution, and reproduction in any medium, provided the original work is properly cited. 
program intervention targeting middle-aged women has been lively. The effect on various physiological variables has been proved. However, there has been no integrated study on a combined exercise program targeting middle-aged women and systematic analysis thereof, and it appears that size and direction of the effect of the combined exercise program on the subordination variables are diverse and inconsistent.

Therefore, this study would like to provide a comprehensive analysis on an exercise program intervention method and psychological variables related thereto through a review of documents which deal with intervention of a combined exercise program targeting middle-aged women and propose the effect of psychological variables.

\section{MATERIALS AND METHODS}

\section{Study design}

This study is meta-analysis which analyzes the effect size (ES) of an exercise program targeting middle-aged women.

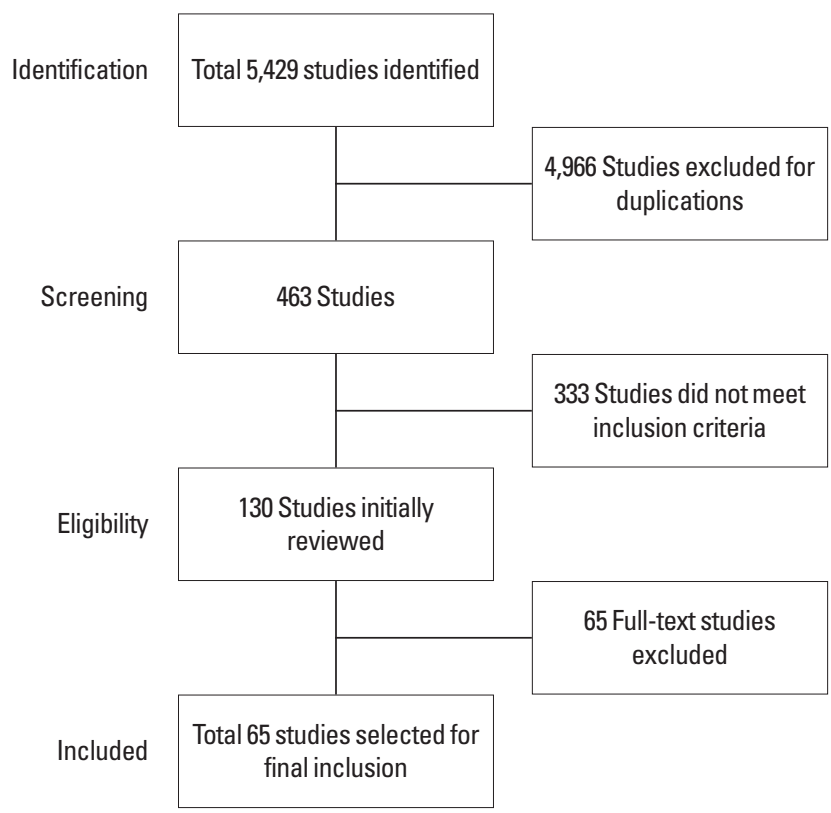

Fig. 1. The method for collecting papers suitable for the purpose of the study is to search the National Assembly Library and the database of Korea Education and Research Information Service, Koreanstudies Information Service System, and Research Information Sharing Service by entering the keywords of "middle-aged women, exercise, blood lipid," "middle-aged women, exercise, hormone," "women, exercise, blood," and "women and combined exercise program." A total of 5,429 papers satisfying the selection criteria were searched and only quantitative studies were selected except for the overlapping papers. A total of 65 papers suitable for the analysis of this study were finally selected.

\section{Reference search and data extraction}

The method for collecting papers suitable for the purpose of the study is to search the National Assembly Library and the database of Korea Education and Research Information Service, Koreanstudies Information Service System, and Research Information Sharing Service by entering the keywords of "middle-aged women, exercise, blood lipid," "middle-aged women, exercise, hormone," "women, exercise, blood," and "women and combined exercise program." A total of 5,429 papers satisfying the selection criteria were searched and only quantitative studies were selected except for the overlapping papers. A total of 65 papers suitable for the analysis of this study were finally selected (Fig. 1).

\section{Statistical analysis}

The ES was calculated using comprehensive meta-analysis and the homogeneity test was performed. The ES was calculated with a random effects model in which, if the homogeneity is not ensured, the weights are reset considering the variation among subjects and the heterogeneity between the studies. The size of the effect was calculated by applying the formula using mean and integrated standard deviation of the experimental group and the control group. As for the analysis result, the number of examples of the ES, size of effect, homogeneity statistical quantity, and 95\% confidence interval $(\mathrm{CI})$ are presented as a table, and statistical significance level of the ES was $P<0.05$, and the $\mathrm{CI}$ was $95 \%$.

\section{RESULTS}

\section{Overall effects}

In this study, the heterogeneity of individual studies was calculated by homogeneity test. Based on the random effect model, the combined exercise program showed the ES of 0.606 , which was a middle or higher level, and thus was statistically significant $(P<0.05)$ (Table 1$)$.

\section{ES of physiological variables of the combined exercise program}

The ES of the physiologically relevant variable group of the combined exercise program was as follows: in case of blood lipid, the ES was middle level or higher in an order of triglyceride

Table 1. Entire effect size of the combined exercise program

\begin{tabular}{lccccc}
\hline & No. & $\begin{array}{c}\text { Effect } \\
\text { size }\end{array}$ & $P$-value & $\begin{array}{c}95 \% \text { Confidence } \\
\text { interval }\end{array}$ & $\begin{array}{c}\text { Standard } \\
\text { error }\end{array}$ \\
\hline Combined exercise & 65 & 0.606 & $<0.001$ & $0.512-0.969$ & 0.048
\end{tabular}


Table 2. Effect size of physiological variables of combined exercise program

\begin{tabular}{lrrrr}
\hline Lower factor & No. & $\begin{array}{c}\text { Effect } \\
\text { size }\end{array}$ & $\begin{array}{c}95 \% \text { Confidence } \\
\text { interval }\end{array}$ & $P$-value \\
\hline Blood lipid profile & & & & \\
Total cholesterol & 46 & 0.509 & $0.329-0.689$ & $<0.001$ \\
Triglyceride & 53 & 0.720 & $0.504-0.936$ & $<0.001$ \\
High density lipoprotein cholesterol & 53 & 0.541 & $0.346-0.735$ & $<0.001$ \\
Low density lipoprotein cholesterol & 42 & 0.658 & $0.399-0.970$ & $<0.001$ \\
$\quad$ Obesity hormone & & & - & \\
$\quad$ Insulin & 22 & 0.229 & $-0.159-0.614$ & 0.244 \\
$\quad$ Leptin & 8 & 0.959 & $0.085-1.832$ & 0.031 \\
$\quad$ Adiponectin & 11 & 0.519 & $-0.048-1.086$ & 0.073 \\
$\quad$ Homeostatic model assessment & 18 & 0.647 & $0.167-1.126$ & 0.008 \\
$\quad$ insulin resistance & & & & \\
Aging hormone & & & - & \\
$\quad$ Estrogen & 5 & 0.811 & $0.243-1.380$ & 0.005 \\
$\quad$ Growth-hormone & 7 & 0.753 & $0.325-1.182$ & 0.001 \\
Inflammatory factors & & & - & \\
$\quad$ C-reactive protein & 12 & 0.492 & $-0.042-1.026$ & 0.071 \\
$\quad$ Tumor necrosis factor-alpha & 9 & 0.795 & $0.258-1.331$ & 0.004 \\
Interleukin-6 & 6 & 0.699 & $0.301-1.098$ & 0.001 \\
\hline
\end{tabular}

(0.720), low-density lipoprotein cholesterol (0.685), high-density lipoprotein cholesterol (0.541), and total cholesterol (0.959). In case of obesity hormone, the ES was greater in Leptin (0.959); homeostatic model assessment-insulin resistance (0.647), and Adiponectin (0.519) showed the ES of a middle level or higher; and Insulin (0.229) showed smaller ES. In the aging hormone group, estrogen (0.811) showed a large ES and growth hormone (0.753) showed a medium or greater ES. As for inflammation factors, tumor necrosis factor- $\alpha$ (0.795), interleukin-6 (0.699) showed a medium or greater ES, and C-reactive protein (CRP) (0.492) showed a medium ES. In all the variables excluding Insulin (CI, -0.15 to 0.614), 0 is not included in the $95 \%$ of $\mathrm{CI}$ and it was statistically significant $(P<0.05)$ (Table 2).

\section{ES according to combined exercise program intervention method}

As a result of calculating the ES according to a combined exercise program intervention method, as for an exercise period, the $\mathrm{ES}$ of a medium or higher level was exhibited in an order of 8 weeks (0.691), 9-12 weeks (0.596), and 13 weeks or more (0.303). In case of an exercise frequency, it showed the ES of a medium level or higher in an order of 2 times a week (0.713), 4 times a week (0.670), 5 times a week (0.658), and 3 times a weeks (0.562). For exercise time, the largest size effect was shown for more than 1.5 to $2 \mathrm{hr}$ (1.081). The medium or higher level of ES was shown for
Table 3. Effect size according to combined exercise program intervention method

\begin{tabular}{lrccc}
\hline Lower factor & No. & Effect size & $\begin{array}{c}95 \% \text { Confidence } \\
\text { interval }\end{array}$ & $P$-value \\
\hline Exercise duration & & & & \\
$\quad 8$ weeks & 44 & 0.691 & $0.477-0.904$ & $<0.001$ \\
9-12 weeks & 245 & 0.596 & $0.492-0.701$ & $<0.001$ \\
13 weeks or more & 7 & 0.303 & $-0.462-1.068$ & 0.438 \\
Exercise frequency & & & - & \\
2 times a week & 6 & 0.713 & $0.474-1.232$ & $<0.001$ \\
3 times a week & 179 & 0.562 & $0.441-0.683$ & $<0.001$ \\
4 times a week & 50 & 0.670 & $0.429-0.911$ & $<0.001$ \\
5 times a week & 54 & 0.658 & $0.434-0.881$ & $<0.001$ \\
Exercise time & & & - & \\
30 min-1 hr & 183 & 0.564 & $0.707-1.455$ & $<0.001$ \\
$1-1.5$ hr & 93 & 0.587 & $0.429-0.745$ & $<0.001$ \\
$1.5-2$ hr & 20 & 1.081 & $0.707-1.455$ & $<0.001$ \\
Exercise intensity & & & - & \\
$40 \%$ or more & 4 & 2.211 & $0.826-3.596$ & $<0.001$ \\
$55 \%$ or more & 148 & 0.652 & $0.524-0.779$ & $<0.001$ \\
$70 \%$ or more & 130 & 0.536 & $0.387-0.685$ & $<0.001$ \\
\hline
\end{tabular}

Table 4. Effect size according to characteristics of study subject of combined exercise program

\begin{tabular}{lrccc}
\hline Lower factor & No. & Effect size & $\begin{array}{c}95 \% \text { Confidence } \\
\text { interval }\end{array}$ & $P$-value \\
\hline Age (yr) & & & & \\
$40-49$ & 150 & 0.719 & $0.586-0.853$ & $<0.001$ \\
$50-59$ & 140 & 0.485 & $0.353-0.617$ & $<0.001$ \\
$60-65$ & 6 & 0.522 & $-0.018-1.063$ & 0.058 \\
Menopause & & & - & \\
$\quad$ No information & 233 & 0.601 & $0.494-0.707$ & $<0.001$ \\
$\quad$ Menopause & 63 & 0.630 & $0.437-0.823$ & $<0.001$ \\
Body fat percentage & & & - & \\
$\quad$ Less than 30\% & 20 & 1.031 & $0.645-1.417$ & $<0.001$ \\
30\% or more & 270 & 0.555 & $0.459-0.651$ & $<0.001$ \\
\hline
\end{tabular}

more than 1 to $1.5 \mathrm{hr}(0.587)$, more than $30 \mathrm{~min}$ to $1 \mathrm{hr}(0.564)$. Exercise intensity showed the largest ES at over 40\% (2.211), more than $55 \%$ (0.652) and more than $70 \%$ (0.536). All the variables except for the exercise period of 13 weeks or more (CI, -0.462 to 1.068$)$ were statistically significant $(P<0.05)$ because they did not include 0 in the $95 \% \mathrm{CI}$ (Table 3).

\section{ES according to characteristics of study of combined exercise program}

The ES of the combined exercise was calculated as specified below: the size of the effect was medium or higher level for $40-49$ 
years old (0.719) and 60-65 years old (0.522) and medium ES for 50-59 years (0.485). According to the presence or absence of menopause, the ES was medium or higher in the order of postmenopausal (0.630) and no information about menopause (0.601). The ES according to body fat percentage showed large ES for body fat less than 30\% (1.031), and the medium or higher level was shown for the body fat percentage which was more than $30 \%$ (0.555). All the variables except 60-65 years (CI, -0.018 to 1.063), 0 is not included in the $\mathrm{CI}$ of $95 \%$ and thus was statistically significant $(P<0.05)$ (Table 4$)$.

\section{DISCUSSION}

The purpose of this study is to investigate the effects of the combined exercise program on the physiological variables of middle-aged women. For this purpose, a meta-analysis of 65 research papers complying with the data selection criteria was conducted for the papers, doctoral theses, and journals published in Korea from 2005 to 2015. The overall ES (0.606) of the combined exercise program was a medium level or higher. Until now, studies regarding various exercise programs targeting middle-aged women have been continuously conducted and analysis results thereof have been integrated and analyzed. It appears that an exercise program has a positive effect on promoting one's health condition.

The aforementioned result of the study is in line with the study (Forbes, 1992; Kligman and Pepin, 1992) mentioning that physiologically variables of the combined exercise program showed a significant ES in most of the blood lipid, obesity hormone group, aging hormone group, and inflammatory factors. This indicates that exercise activates metabolism and thus aging may not be completed avoided, but progress speed of aging can be delayed and immune reaction can be affected. The CRP of insulin, adiponectin, and inflammatory factors of the obesity hormone group was not statistically significant. This indicates that the change of physiological variables due to exercise varies with the type of exercise, exercise time, exercise intensity and exercise frequency, and this is understood as a mechanism of adaption to physical stress and is related to reaction mechanism with respect to exercise (Kjaer, 1989). Here, it should be considered that the methods of intervention and other environmental factors are not the same for each study.

As a result of the subanalysis according to the intervention method, it was found that the conditions which result in the most effective effect of the combined exercise program were more than 8 weeks, 2 times a week, 1.5 to $2 \mathrm{hr}$, and $40 \%$ intensity. In the fre- quency of exercise, the ESs was at a middle or higher level for all the frequencies in the order of twice a week (0.713), 4 times a week (0.670), 5 times a week (0.658), and 3 times a week (0.562). Most of the combined exercise programs had the highest number of exercises 3 times a week, but the result shows that the exercise has the effect with only exercise of 2 times a week. The duration of exercise showed the highest ES for 1.5 to $2 \mathrm{hr}$. The results of this study showed that during the 120-min combined exercise program, the exercise time was gradually extended and the exercise was conducted in a stable scope. The result showed a statistically significant effect and the effect (1.081) was high in the present study. Exercise intensity showed a high ES at $40 \%$ or more intensity, but the number of cases was four only and thus, objectivity of the result was not that high. Therefore, caution is needed in interpretation of this result. It is thought that it is more important to exercise according to the individual's ability in both low intensity exercise and high intensity exercise as the ES is more than medium in both exercise frequency of $55 \%$ or more and $70 \%$ or more. It seems preferable to increase the effect through the exercise with gradually increasing intensity. According to the American College of Sports Medicine, it is recommended that exercise intensity is $40 \%-70 \%$, exercise time is $45-60 \mathrm{~min}$, total energy consumption is $300-400 \mathrm{kcal}$ per day. It was said that consumption of calories more than $900 \mathrm{kcal}$ for one week will bring a gradual change and thus seems effect. This is consistent with the result of this study.

This study confirmed that the combined exercise program targeting middle-aged women showed the ES of a medium level or higher for blood lipid, obesity hormone, aging hormone, and inflammatory factors, exhibiting a positive change especially to psychological variables. It was found that the combined exercise program is a very effective intervention method to overcome functional loss and weakened body function of middle-age women by preventing physical strength from being degraded and hormones from decreasing. In this study, the effect of the characteristics of a study subject and the characteristics of exercise intervention on the exercise program has been analyzed in a comprehensive manner and a direction for an exercise program is proposed. It is believed that this study can reduce inefficiencies of overly redundant studies regarding the effect of a combined exercise program targeting middle-aged women, and provide the grounds for the basic work for the integrated classification of the physiological variables associated with the effect of an exercise program. 


\section{CONFLICT OF INTEREST}

No potential conflict of interest relevant to this article was reported.

\section{REFERENCES}

Andersen RE, Wadden TA, Bartlett SJ, Vogt RA, Weinstock RS. Relation of weight loss to changes in serum lipids and lipoproteins in obese women. Am J Clin Nutr 1995;62:350-357.

Brehm BA. Elevation of metabolic rate following exercise. Implications for weight loss. Sports Med 1988;6:72-78.

Bruunsgaard H, Pedersen BK. Special feature for the Olympics: effects of exercise on the immune system: effects of exercise on the immune system in the elderly population. Immunol Cell Biol 2000;78:523-531.

Dwarakanathan A. Diabetes update. J Insur Med 2006;38:20-30.

Forbes EJ. Exercise: wellness maintenance for the elderly client. Holist Nurs Pract 1992;6:14-22.

Gleeson M, McDonald WA, Pyne DB, Clancy RL, Cripps AW, Francis JL, Fricker PA. Immune status and respiratory illness for elite swimmers during a 12-week training cycle. Int J Sports Med 2000;21:302-307.

Huang G, Osness WH. Changes in pulmonary function response to a 10week controlled exercise program in sedentary elderly adults. Percept Mot Skills 2005;100:394-402.

Kiefer I, Kunze U, Mitsche N, Kunze M. Obesity in Austria: epidemiologic and social medicine aspects. Acta Med Austriaca 1998;25:126-128.

Kjaer M. Epinephrine and some other hormonal responses to exercise in man: with special reference to physical training. Int J Sports Med 1989; 10:2-15.

Kligman EW, Pepin E. Prescribing physical activity for older patients. Geriatrics 1992;47:33-34, 37-44, 47.

Lakier Smith L. Overtraining, excessive exercise, and altered immunity: is this a T helper-1 versus T helper-2 lymphocyte response? Sports Med 2003;33:347-364.

Orsini N, Bellocco R, Bottai M, Pagano M, Wolk A. Age and temporal trends of total physical activity among Swedish women. Med Sci Sports Exerc 2006;38:240-245.

Poehlman ET, Viers HF, Detzer M. Influence of physical activity and dietary restraint on resting energy expenditure in young nonobese females. Can J Physiol Pharmacol 1991;69:320-326. 